\title{
Tetrahedron Equation and Cyclic Quantum Dilogarithm Identities
}

\section{Andrei Bytsko ${ }^{1,2}$ and Alexander Volkov ${ }^{1,2}$}

${ }^{1}$ Section of Mathematics, University of Geneva, C.P. 64, 1211 Genève 4, Switzerland and ${ }^{2}$ Steklov Mathematical Institute, Fontanka 27, 191023

St. Petersburg, Russia

Correspondence to be sent to: andrey.bytsko@unige.ch

We establish a hierarchy of quantum dilogarithm identities associated to a sequence of triangular shaped quivers. The tetrahedron equation plays a key role in our construction.

\section{Introduction. Main Results}

Fix $q \in(0,1)$. The quantum exponential function is the following formal series:

$$
\langle x\rangle_{q}=\sum_{n=0}^{\infty} \frac{(-x)^{n}}{(1-q) \cdots\left(1-q^{n}\right)} .
$$

Its key functional property is the equation $\langle q x\rangle_{q}=(1+x)\langle x\rangle_{q}$ due to which the quantum exponential function appears naturally, in particular, in the theory of cluster algebras.

It is well known that if $\mathrm{X}$ and $\mathrm{Y}$ are two $q$-commuting indeterminates, that is they satisfy the commutation relation

$$
\mathrm{YX}=q \mathrm{XY}
$$


then the following identities hold:

$$
\begin{aligned}
\langle\mathrm{X}\rangle_{q}\langle\mathrm{Y}\rangle_{q} & =\langle\mathrm{X}+\mathrm{Y}\rangle_{q}, \\
\langle\mathrm{X}\rangle_{q}\langle\mathrm{XY}\rangle_{q}\langle\mathrm{Y}\rangle_{q} & =\langle\mathrm{Y}\rangle_{q}\langle\mathrm{X}\rangle_{q} .
\end{aligned}
$$

The first one is due to Schützenberger [12]. The second identity was found in [3] and is now commonly called the pentagon identity. It is also often called a quantum dilogarithm identity because it is closely related to the five-term dilogarithm identity [3, 4, 14].

Now take three pairwise $q$-commuting indeterminates $\mathrm{X}, \mathrm{Y}$, and $\mathrm{Z}$,

$$
\mathrm{YX}=q \mathrm{XY}, \quad \mathrm{XZ}=q \mathrm{ZX}, \quad \mathrm{ZY}=q \mathrm{YZ} .
$$

Following $[9,13]$, we utilize the pentagon relation twice and find that

$$
\langle\mathrm{X}\rangle_{q}\langle\mathrm{Z}\rangle_{q}\langle\mathrm{XY}\rangle_{q}\langle\mathrm{Y}\rangle_{q}=\langle\mathrm{Z}\rangle_{q}\langle\mathrm{ZXX}\rangle_{q}\langle\mathrm{X}\rangle_{q}\langle\mathrm{XY}\rangle_{q}\langle\mathrm{Y}\rangle_{q}=\langle\mathrm{Z}\rangle_{q}\langle\mathrm{ZX}\rangle_{q}\langle\mathrm{Y}\rangle_{q}\langle\mathrm{X}\rangle_{q}
$$

Note that $X Y$ and $Z$ commute. Permuting $\langle X Y\rangle_{q}$ and $\langle Z\rangle_{q}$ on the l.h.s., we bring identity (6) to the form $\mathbf{T}=\rho(\mathrm{T})$, where $\mathbf{T}=\langle\mathbf{X}\rangle_{q}\langle\mathbf{X Y}\rangle_{q}\langle\mathbf{Z}\rangle_{q}\langle\mathrm{Y}\rangle_{q}$ and $\rho: \mathrm{X} \rightarrow \mathbf{Z}, \mathbf{Y} \rightarrow \mathbf{X}, \mathbf{Z} \rightarrow \mathbf{Y}$ is an order 3 automorphism of the associative algebra defined by presentation (5). Hence follows immediately the following triple identity:

$$
\mathbf{T}=\rho(\mathbf{T})=\rho(\rho(\mathbf{T})),
$$

or, explicitly,

$$
\langle\mathrm{X}\rangle_{q}\langle\mathrm{XY}\rangle_{q}\langle\mathrm{Z}\rangle_{q}\langle\mathrm{Y}\rangle_{q}=\langle\mathrm{Z}\rangle_{q}\langle\mathrm{ZX}\rangle_{q}\langle\mathrm{Y}\rangle_{q}\langle\mathrm{X}\rangle_{q}=\langle\mathrm{Y}\rangle_{q}\langle\mathrm{YZ}\rangle_{q}\langle\mathrm{X}\rangle_{q}\langle\mathrm{Z}\rangle_{q}
$$

In view of (7), we will say that (8) is a cyclic quantum dilogarithm identity.

The goal of this paper is to obtain a hierarchy of cyclic quantum dilogarithm identities in which (8) would be the first nontrivial member. For this purpose, we will introduce an algebra $\mathcal{T}_{N}$ with generators assigned to the vertices of a certain quiver $Q_{N}$. In what follows, $N$ stands for an integer number $>1$.

Definition 1.1. The quiver $Q_{N}$ is an oriented graph with vertices that are labeled by pairs of integer numbers $(i, j)$ such that $1 \leq i<j \leq N$. The directed edges go from $(i, j)$ to $(i, j+1)$, from $(i, j)$ to $(i+1, j)$, and from $(i+1, j+1)$ to $(i, j)$. 
Thus, $Q_{N}$ has $\left(\begin{array}{c}N \\ 2\end{array}\right)$ vertices. For instance, the quivers $Q_{3}$ and $Q_{4}$ are
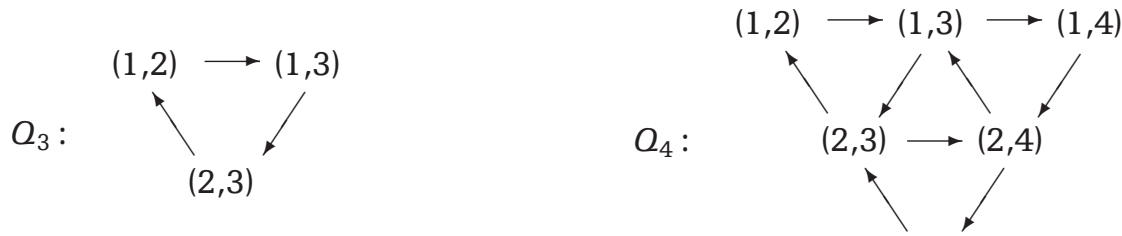

$(3,4)$

Given $Q_{N}$, one defines in the standard way its skew-symmetric incidence matrix $B: B_{(i, j),\left(i^{\prime}, j^{\prime}\right)}=-B_{\left(i^{\prime}, j^{\prime}\right),(i, j)}=1$ if there is a directed edge going from $(i, j)$ to $\left(i^{\prime}, j^{\prime}\right)$, and $B_{(i, j),\left(i^{\prime}, j^{\prime}\right)}=0$ if the vertices $(i, j)$ and $\left(i^{\prime}, j^{\prime}\right)$ are not connected.

Definition 1.2. The algebra $\mathcal{T}_{N}$ associated with the quiver $Q_{N}$ is a unital associative algebra over $\mathbb{R}$ with $\left(\begin{array}{c}N \\ 2\end{array}\right)$ generators $Z_{i j}, 1 \leq i<j \leq N$, and the following defining relations:

$$
\mathrm{Z}_{i j} \mathrm{Z}_{i^{\prime} j^{\prime}}=q^{B_{(i, j),\left(i^{\prime}, j^{\prime}\right)}} \mathbf{Z}_{i^{\prime} j^{\prime}} \mathbf{Z}_{i j}
$$

In particular, $\mathcal{T}_{2}$ is generated by a single generator. The algebra $\mathcal{T}_{3}$ (with generators $Z_{12}, Z_{13}, Z_{23}$ renamed $X, Z, Y$ ) coincides with the algebra defined by presentation (5).

Remark 1.3. Let $I$ be a subset of vertices of $Q_{N}$. Consider a linear homomorphism $\sigma_{I}$ such that $\sigma_{I}\left(Z_{i j}\right)=0$ if $(i, j) \in I$ and $\sigma_{I}\left(Z_{i j}\right)=Z_{i j}$ otherwise. Since relations (9) are homogeneous, $\sigma_{I}$ is an algebra homomorphism from $\mathcal{T}_{N}$ to its subalgebra. In particular, sending $Z_{i N}$ to zero for all $i$, we reduce $\mathcal{T}_{N}$ to $\mathcal{T}_{N-1}$. Thus, we have a chain of subalgebra inclusions: $\mathcal{T}_{2} \subset \mathcal{T}_{3} \cdots \subset \mathcal{T}_{N-1} \subset \mathcal{T}_{N}$

Remark 1.4. The center $\mathcal{Z}_{N}$ of the algebra $\mathcal{T}_{N}$ has dimension $N / 2$ if $N$ is even and $(N-1) / 2$ if $N$ is odd. (See Section 2.2 for more details.)

The quiver $Q_{N}$ is mapped to itself by a clockwise rotation about its geometric center by $2 \pi / 3$. Hence, the algebra $\mathcal{T}_{N}$ admits an automorphism $\rho$ of order 3 ,

$$
\rho\left(Z_{i j}\right)=Z_{j-i, N+1-i}
$$

Furthermore, the quiver $Q_{N}$ is mapped into a dual quiver (where all the arrows are reverted) by a reflection with respect to either of its three axes of symmetry passing through the corner vertices. Hence, the algebra $\mathcal{T}_{N}$ admits three involutive 
anti-automorphisms:

$$
\mu_{1}\left(Z_{i j}\right)=Z_{j-i, j}, \quad \mu_{2}\left(Z_{i j}\right)=Z_{N+1-j, N+1-i}, \quad \mu_{3}\left(Z_{i j}\right)=Z_{i, N+1+i-j}
$$

Note that

$$
\mu_{1} \circ \mu_{2}=\mu_{2} \circ \mu_{3}=\mu_{3} \circ \mu_{1}=\rho .
$$

Let us adopt the following notations for products of noncommuting factors. Let $\prec$ be the lexicographic order relation of elements of a set $\Lambda \subset \mathbb{Z}^{m}$. Then $\vec{\prod}_{\lambda \in \Lambda} f_{\lambda}$ and $\overleftarrow{\prod}_{\lambda \in \Lambda} f_{\lambda}$ stand for ordered products, where $f_{\lambda}$ is put to the right (respectively, to the left) of all $f_{\lambda^{\prime}}$ such that $\lambda^{\prime} \prec \lambda$. In particular, if $\Lambda=[1, k] \subset \mathbb{Z}$, then $\vec{\prod}_{\lambda \in \Lambda} f_{\lambda}=f_{1} \cdots f_{k}$ and $\overleftarrow{\prod}_{\lambda \in \Lambda} f_{\lambda}=f_{k} \cdots f_{1}$

Let $\Lambda_{N} \subset \mathbb{Z}^{3}$ be the following discrete tetrahedron containing $\left(\begin{array}{c}N+1 \\ 3\end{array}\right)$ points:

$$
\Lambda_{N}=\{\lambda=(a, b, c) \mid 1 \leq a<b<c \leq N+1\} .
$$

To each point $\lambda=(a, b, c) \in \Lambda_{N}$, we associate the following element of $\mathcal{T}_{N}$ (strictly speaking, of its completion):

$$
\mathbb{R}_{\lambda}=\left\langle\prod_{0 \leq k \leq c-b-1} \mathrm{Z}_{a+k, b+k}\right\rangle_{q}
$$

Now we can define an analog of the element T used in (7).

Definition 1.5. $\mathrm{T}_{N} \in \mathcal{T}_{N}$ is the following lexicographically ordered product:

$$
\mathrm{T}_{N}=\prod_{\lambda \in \Lambda_{N}} \mathbb{R}_{\lambda}
$$

In particular, we have $T_{2}=\mathbb{R}_{123}=\left\langle Z_{12}\right\rangle_{q}$ and $T_{3}=\mathbb{R}_{123} \mathbb{R}_{124} \mathbb{R}_{134} \mathbb{R}_{234}=\left\langle Z_{12}\right\rangle_{q}$ $\left\langle Z_{12} Z_{23}\right\rangle_{q}\left\langle Z_{13}\right\rangle_{q}\left\langle Z_{23}\right\rangle_{q}$

Remark 1.6. Some factors in the product (15) mutually commute, as, for example, $\mathbb{R}_{124}$ and $\mathbb{R}_{134}$ in $\mathrm{T}_{3}$. Therefore, $\mathrm{T}_{N}$ admits a number of equivalent expressions obtained by permutations of such factors. Some of these expressions are given in Lemma 2.7.

We will say that a quantum dilogarithm identity is of the type $n \sim m$ if it involves $n$ quantum exponentials with monomial arguments on one side and $m$ on the other. For instance, (4) and (8) are of the type 2-3 and 4-4, respectively. The main result 
of the present work is a family of cyclic quantum dilogarithm identities of the type $\left(\begin{array}{c}N+1 \\ 3\end{array}\right) \sim\left(\begin{array}{c}N+1 \\ 3\end{array}\right)$.

Theorem 1.7. Identities

$$
\begin{aligned}
& \mathbf{T}_{N}=\mu_{1}\left(\mathbf{T}_{N}\right)=\mu_{2}\left(\mathbf{T}_{N}\right)=\mu_{3}\left(\mathbf{T}_{N}\right), \\
& \mathbf{T}_{N}=\rho\left(\mathbf{T}_{N}\right)=\rho\left(\rho\left(\mathbf{T}_{N}\right)\right),
\end{aligned}
$$

hold for any integer $N \geq 2$.

Remark 1.8. Identities (16) involve the anti-automorphism transformations $\mu_{a}$ corresponding to a reflection rather than a rotation symmetry of the quiver. Nevertheless, two of these identities are almost cyclic in the following sense. We will see below (cf. Lemma A.2) that $\mu_{2}$ applied to $T_{N}$ acts almost as an identical transformation just permuting some commuting quantum exponentials. (As an example, apply to (8) an antiautomorphism that maps $Z$ to itself and exchanges $X$ and $Y$.) Along with (12) this implies that, again up to a permutation of commuting factors, $\mu_{1}$ applied to $T_{N}$ acts as $\rho$, and $\mu_{3}$ applied to $\mathrm{T}_{N}$ acts as $\rho^{-1}=\rho \circ \rho$.

It is clear from the definition (1) of the quantum exponential that $\langle 0\rangle_{q}=1$. This, along with Remark 1.3, implies the following proposition.

Proposition 1.9. Let $I$ be a subset of the set of vertices of $Q_{N}$. In the identity (17), replace with unity every quantum exponential that contains at least one $Z_{i j}$ with $(i, j) \in I$. The result is a correct quantum dilogarithm identity.

Note that the resulting reduced identity is not necessarily cyclic. For instance, sending $Z$ to 0 in the $4-4$ identity (8), we obtain the pentagon identity (4).

Quantum dilogarithm identities associated with various quivers have close connections with (quantum) cluster algebras and $Y$-systems; see, for example, [1, 5, 8, 10]. Quantum torus algebras of which $\mathcal{T}_{N}$ is a particular example play an important role in this context. Within the cluster algebras framework, a classical or a quantum dilogarithm identity is a consequence of existence of a periodic sequence of mutations of a quiver and seeds assigned to the quiver [10, 11]. Examples of dilogarithm identities obtained by this approach for (direct products of) Dynkin quivers are given in [7, 10]. Identities given in Theorem $1.7 \mathrm{can}$ in principle be obtained by the quiver mutation method as well. But in the present paper, we will derive them by a different representation theoretic approach related to inversions of maximal chains of higher Bruhat orders. 
Our motivation for studying a specific family of quantum dilogarithm identities is an observation that they are accessible from the 4-4 identities (8). For instance, let us demonstrate how to derive the equality $T_{4}=\rho\left(T_{4}\right)$ without invoking the pentagon relation but using only the 4-4 identities (8):

$$
\begin{aligned}
& \mathrm{T}_{4}=\left\langle\mathrm{Z}_{12}\right\rangle_{q}\left\langle\mathrm{Z}_{12} \mathrm{Z}_{23}\right\rangle_{q}\left\langle\mathrm{Z}_{12} \mathrm{Z}_{23} \mathrm{Z}_{34}\right\rangle_{q}\left\langle\mathrm{Z}_{13}\right\rangle_{q}\left\langle\mathrm{Z}_{13} \mathrm{Z}_{24}\right\rangle_{q}\left\langle\mathrm{Z}_{14}\right\rangle_{q}\left\langle\mathrm{Z}_{23}\right\rangle_{q}\left\langle\mathrm{Z}_{23} \mathrm{Z}_{34}\right\rangle_{q}\left\langle\mathrm{Z}_{24}\right\rangle_{q}\left\langle\mathrm{Z}_{34}\right\rangle_{q} \\
& =\underline{\left\langle Z_{12}\right\rangle_{q}\left\langle Z_{12} Z_{23}\right\rangle_{q}\left\langle Z_{13}\right\rangle_{q}\left\langle Z_{23}\right\rangle_{q}}\left\langle Z_{12} Z_{23} Z_{34}\right\rangle_{q}\left\langle Z_{13} Z_{24}\right\rangle_{q}\left\langle Z_{23} Z_{34}\right\rangle_{q}\left\langle Z_{14}\right\rangle_{q}\left\langle Z_{24}\right\rangle_{q}\left\langle Z_{34}\right\rangle_{q} \\
& =\left\langle Z_{13}\right\rangle_{q}\left\langle Z_{13} Z_{12}\right\rangle_{q}\left\langle Z_{23}\right\rangle_{q}\left\langle Z_{12}\right\rangle_{q}\left\langle Z_{12} Z_{23} Z_{34}\right\rangle_{q}\left\langle Z_{13} Z_{24}\right\rangle_{q}\left\langle Z_{23} Z_{34}\right\rangle_{q}\left\langle Z_{14}\right\rangle_{q}\left\langle Z_{24}\right\rangle_{q}\left\langle Z_{34}\right\rangle_{q} \\
& =\left\langle Z_{13}\right\rangle_{q}\left\langle Z_{23}\right\rangle_{q}\left\langle Z_{13} Z_{12}\right\rangle_{q}\left\langle Z_{13} Z_{24}\right\rangle_{q}\left\langle Z_{13} Z_{24} Z_{12}\right\rangle_{q}\left\langle Z_{23} Z_{34}\right\rangle_{q}\left\langle Z_{12}\right\rangle_{q}\left\langle Z_{14}\right\rangle_{q}\left\langle Z_{24}\right\rangle_{q}\left\langle Z_{34}\right\rangle_{q} \\
& =\left\langle Z_{13}\right\rangle_{q}\left\langle Z_{23}\right\rangle_{q}\left\langle Z_{13} Z_{24}\right\rangle_{q}\left\langle Z_{13} Z_{12}\right\rangle_{q}\left\langle Z_{13} Z_{12} Z_{24}\right\rangle_{q}\left\langle Z_{14}\right\rangle_{q}\left\langle Z_{24}\right\rangle_{q}\left\langle Z_{23} Z_{34}\right\rangle_{q}\left\langle Z_{34}\right\rangle_{q}\left\langle Z_{12}\right\rangle_{q} \\
& =\left\langle Z_{13}\right\rangle_{q}\left\langle Z_{23}\right\rangle_{q}\left\langle Z_{13} Z_{24}\right\rangle_{q}\left\langle Z_{14}\right\rangle_{q}\left\langle Z_{14} Z_{13} Z_{12}\right\rangle_{q}\left\langle Z_{24}\right\rangle_{q}\left\langle Z_{13} Z_{12}\right\rangle_{q}\left\langle Z_{23} Z_{34}\right\rangle_{q}\left\langle Z_{34}\right\rangle_{q}\left\langle Z_{12}\right\rangle_{q} \\
& =\left\langle Z_{13}\right\rangle_{q}\left\langle Z_{13} Z_{24}\right\rangle_{q}\left\langle Z_{14}\right\rangle_{q}\left\langle Z_{14} Z_{13} Z_{12}\right\rangle_{q}\left\langle Z_{23}\right\rangle_{q}\left\langle Z_{23} Z_{34}\right\rangle_{q}\left\langle Z_{24}\right\rangle_{q}\left\langle Z_{34}\right\rangle_{q}\left\langle Z_{13} Z_{12}\right\rangle_{q}\left\langle Z_{12}\right\rangle_{q}
\end{aligned}
$$

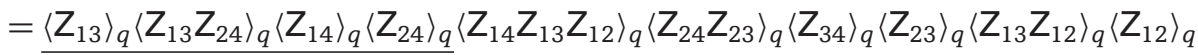

$$
\begin{aligned}
& =\left\langle Z_{14}\right\rangle_{q}\left\langle Z_{14} Z_{13}\right\rangle_{q}\left\langle Z_{24}\right\rangle_{q}\left\langle Z_{13}\right\rangle_{q}\left\langle Z_{14} Z_{13} Z_{12}\right\rangle_{q}\left\langle Z_{24} Z_{23}\right\rangle_{q}\left\langle Z_{34}\right\rangle_{q}\left\langle Z_{13} Z_{12}\right\rangle_{q}\left\langle Z_{23}\right\rangle_{q}\left\langle Z_{12}\right\rangle_{q} \\
& =\left\langle Z_{14}\right\rangle_{q}\left\langle Z_{14} Z_{13}\right\rangle_{q}\left\langle Z_{14} Z_{13} Z_{12}\right\rangle_{q}\left\langle Z_{24}\right\rangle_{q}\left\langle Z_{24} Z_{23}\right\rangle_{q}\left\langle Z_{34}\right\rangle_{q}\left\langle Z_{13}\right\rangle_{q}\left\langle Z_{13} Z_{12}\right\rangle_{q}\left\langle Z_{23}\right\rangle_{q}\left\langle Z_{12}\right\rangle_{q} \\
& =\rho\left(\mathbf{T}_{4}\right) \text {. }
\end{aligned}
$$

The underlined terms were transformed by applying the 4-4 identities (8). The remaining transformations changed only the order of commuting factors or the order of commuting generators in the arguments of quantum exponentials.

We will give a proof of the identities listed in Theorem 1.7 which makes it evident that they are accessible from the 4-4 identities (8) for all $N$. The origin of this accessibility is that these identities stem from identities for certain words in a group whose generators satisfy the tetrahedron equation:

$$
R_{a b c} R_{a b d} R_{a c d} R_{b c d}=R_{b c d} R_{a c d} R_{a b d} R_{a b c}
$$

The paper is organized as follows. Section 2 contains auxiliary statements which we need to combine together in order to prove Theorem 1.7. Namely, in Section 2.1 we consider families of groups $\mathcal{B}(n, N)$ whose generators satisfy the Yang-Baxter equation (for $n=2$ ), the tetrahedron equation (for $n=3$ ), or their higher analogs (for $n \geq 4$ ). The two 
key technical results here are an identity for certain words containing all the generators of the group $\mathcal{B}(n, N)$ and relation of these words to the element $\mathrm{T}_{N}$. In Section 2.2, we describe the center of $\mathcal{T}_{N}$. In Section 2.3, we consider a local tensor space representation $\phi$ for $\mathcal{B}(3, N)$. In Section 2.4, we explain how evaluation of the above-mentioned identity for words of $\mathcal{B}(3, N)$ in the representation $\phi$ yields ultimately the desired quantum dilogarithm identities for an arbitrary $N$. Appendix contains proofs of all statements given in Section 2.

\section{Main Technical Ingredients}

Below we assume that $N$ and $n$ are positive integers and $N \geq n$.

\subsection{A group with $n$-simplex relations}

Definition 2.1. $\mathcal{B}(n, N)$ is a group with $\left(\begin{array}{l}N \\ n\end{array}\right)$ generators $R_{a_{1}, \ldots, a_{n}}$, where $1 \leq a_{1}<a_{2}<\cdots<$ $a_{n} \leq N$. The group is defined by the following presentation:

(1) The generators commute,

$$
R_{a_{1}, \ldots, a_{n}} R_{b_{1}, \ldots, b_{n}}=R_{b_{1}, \ldots, b_{n}} R_{a_{1}, \ldots, a_{n}}
$$

unless the set $\left\{a_{1}, \ldots, a_{n}\right\} \bigcap\left\{b_{1}, \ldots, b_{n}\right\}$ contains exactly $(n-1)$ element.

(2) If $N>n$, the generators satisfy the following $\left(\begin{array}{c}N \\ n+1\end{array}\right)$ relations:

$$
\prod_{1 \leq j \leq n+1} R_{a_{1}, \ldots, \check{a}_{j}, \ldots, a_{n+1}}=\prod_{1 \leq j \leq n+1}^{\overleftarrow{ }} R_{a_{1}, \ldots, \check{a}_{j}, \ldots, a_{n+1}}
$$

where $\check{a}_{j}$ is dropped.

For $n=1$, relations (20) imply commutativity, $R_{a} R_{b}=R_{b} R_{a}$, so that $\mathcal{B}(1, N)$ is an abelian group with $N$ generators.

For $n=2$, relations (20) have the form of the Yang-Baxter equation,

$$
R_{a b} R_{a c} R_{b c}=R_{b c} R_{a c} R_{a b}, \quad a<b<c,
$$

and $R_{a b}$ commutes with $R_{a^{\prime} b^{\prime}}$ if they have no common index. 
For $n=3$, relations (20) have the form of the tetrahedron equation,

$$
R_{a b c} R_{a b d} R_{a c d} R_{b c d}=R_{b c d} R_{a c d} R_{a b d} R_{a b c}, \quad a<b<c<d,
$$

and $R_{a b c}$ commutes with $R_{a^{\prime} b^{\prime} c^{\prime}}$ unless they have exactly two common indices.

Recall that, given a set $\Lambda \subset \mathbb{Z}^{m}$, we use the symbol $\vec{\prod}_{\lambda \in \Lambda} f_{\lambda}$ to denote the lexicographically ordered product of noncommuting factors.

Definition 2.2. The word $W(n, N) \in \mathcal{B}(n, N)$ is the lexicographically ordered product of all generators of $\mathcal{B}(n, N)$, that is,

$$
W(n, N)=\vec{\prod}_{1 \leq a_{1}<a_{2} \cdots<a_{n} \leq N} R_{a_{1}, \ldots, a_{n}} .
$$

In particular, we have $W(n, n)=R_{1, \ldots, n}$ for all $n$ and $W(1, N)=R_{1} \cdots R_{N}$ for all $N$.

Let $\prec^{\prime}$ be the colexicographic order relation of elements of a set $\Lambda \subset \mathbb{Z}^{m}$. That is, components of elements of $\Lambda$ are compared starting from the right. For instance, $(a, b) \prec^{\prime}(c, d)$ iff $b<d$ or $b=d$ and $a<c$. We will denote by $\vec{\prod}_{\lambda \in \Lambda}^{\prime} f_{\lambda}$ and $\overleftarrow{\prod}_{\lambda \in \Lambda}^{\prime} f_{\lambda}$ ordered products where $f_{\lambda}$ is put to the right (respectively, to the left) of all $f_{\lambda^{\prime}}$ such that $\lambda^{\prime} \prec^{\prime} \lambda$.

Lemma 2.3. Define

$$
W^{\prime}(n, N)=\underset{1 \leq a_{1}<a_{2} \cdots<a_{n} \leq N}{\overrightarrow{\prod_{a}, \ldots, a_{n}}}
$$

Then we have the equality

$$
W(n, N)=W^{\prime}(n, N)
$$

For instance, (23) yields $W(2,4)=R_{12} R_{13} R_{14} R_{23} R_{24} R_{34}$ while (24) yields $W^{\prime}(2,4)=$ $R_{12} R_{13} R_{23} R_{14} R_{24} R_{34}$. These two words coincide since, by (19), $R_{14}$ and $R_{23}$ commute. It is also true in general that one needs to use only the commutativity relations (19) in order to change the order of factors in (24) to match that in (23).

Let $\eta$ be an involutive anti-automorphism of $\mathcal{B}(n, N)$ such that

$$
\eta\left(R_{a_{1}, \ldots, a_{n}}\right)=R_{a_{1}, \ldots, a_{n}}
$$

for all the generators of $\mathcal{B}(n, N)$. Define

$$
\bar{W}(n, N)=\eta(W(n, N)),
$$


which is a word with the order of factors reverse to that of $W(n, N)$. Our first key technical statement is the following theorem.

Theorem 2.4. For all $N \geq n$, we have the equality

$$
W(n, N)=\bar{W}(n, N)
$$

Remark 2.5. A simple inspection of the proof given in the Appendix shows that the invertibility of $R^{\prime}$ s is not really needed. Thus, Theorem 2.4 holds also if $\mathcal{B}(n, N)$ is a semigroup.

Remark 2.6. The presented proof provides a constructive recursive procedure that transforms $W(n, N)$ into $\bar{W}(n, N)$. The transformation involves trivial moves based on (19) and moves $\mathcal{R}_{a_{1}, \ldots, a_{n+1}}$ which transform the 1.h.s. of (20) into its r.h.s. For instance, $\mathcal{R}_{123}(W(2,4))=R_{23} R_{13} R_{12} R_{14} R_{24} R_{34}$. Let $\mathcal{W}(n, N)$ and $\overline{\mathcal{W}}(n, N)$ stand for the compositions of such moves in which $\mathcal{R}$ 's are ordered in the same way as $R^{\prime}$ s are ordered in $W(n, N)$ and $\bar{W}(n, N)$. A simple inspection of the proof shows that the l.h.s. of (28) is transformed into its r.h.s. by $\mathcal{W}^{*}(n+1, N)$, where the star means that trivial moves are included when necessary. Moreover, the same transformation is achieved by $\overline{\mathcal{W}}^{*}(n+1, N)$ if we start each reordering not from the left but from the right. Thus, the moves satisfy the identity $\mathcal{W}^{*}(n, N)=\overline{\mathcal{W}}^{*}(n, N)$ analogous to (28). This identity is closely related to the consistency condition for inversions of maximal chains of higher Bruhat orders; see, for example, [2].

Equations (23) and (24) for $n=2$ and $n=3$ read

$$
\begin{aligned}
& W(2, N)=\prod_{1 \leq a<b \leq N} R_{a b}, \quad W^{\prime}(2, N)=\vec{\prod}_{1 \leq a<b \leq N}^{\prime} R_{a b}, \\
& W(3, N)=\prod_{1 \leq a<b<c \leq N} R_{a b c}, \quad W^{\prime}(3, N)=\vec{\prod}_{1 \leq a<b<c \leq N}^{\prime} R_{a b c} .
\end{aligned}
$$

Recall that $\mathbb{R}_{a b c}$ was defined in (14). We introduce also the following elements of $\mathcal{T}_{N}$ :

$$
\mathbb{R}_{a b}=\prod_{b+1 \leq c \leq N+1} \mathbb{R}_{a b c}
$$


Let us introduce the following homomorphisms from $\mathcal{B}(2, N)$ and $\mathcal{B}(3, N+1)$ to $\mathcal{T}_{N}$ (it should be stressed that they are not algebra homomorphisms):

$$
\sharp: R_{a b} \rightarrow \mathbb{R}_{a b}, \quad \star: R_{a b c} \rightarrow \mathbb{R}_{a b c}, \quad \star \star: R_{a b c} \rightarrow \mathbb{R}_{a, c+a-b, c} .
$$

Lemma 2.7. The element $\mathrm{T}_{N}$ defined in (15) can be obtained as follows:

$$
\begin{gathered}
\mathrm{T}_{N}=(W(2, N))^{\sharp}=\left(W^{\prime}(2, N)\right)^{\sharp}, \\
\mathrm{T}_{N}=(W(3, N+1))^{\star}=\left(W^{\prime}(3, N+1)\right)^{\star}, \\
\mathrm{T}_{N}=(W(3, N+1))^{\star \star}=\left(W^{\prime}(3, N+1)\right)^{\star \star} .
\end{gathered}
$$

This observation suggests that identities (28) can be recast in some quantum dilogarithm identities. Furthermore, Remark 2.6 indicates that one needs to use the 4-4 relation (8) $\left(\begin{array}{c}N+1 \\ 4\end{array}\right)$ times in order to derive identity (17) for given $N$.

\subsection{The center of $\mathcal{T}_{N}$}

Let us assign to each vertex $(i, j)$ of the quiver $Q_{N}$ a nonnegative integer weight $\alpha_{i j}$. Let $\alpha$ denote the vector composed of those weights taken in the lexicographic order. For instance, $\alpha=\left(\alpha_{12}, \alpha_{13}, \alpha_{23}\right)$ if $N=3$.

Monomials

$$
\mathrm{M}(\alpha)=\overrightarrow{\prod_{1 \leq i<j \leq N}} \mathrm{Z}_{i j}^{\alpha_{i j}}, \quad \alpha \in \mathbb{Z}_{\geq 0}^{N(N-1) / 2}
$$

constitute a basis of $\mathcal{T}_{N}$. We will say that $\alpha_{i j}$ are the weights of $\mathrm{M}(\alpha)$.

The algebra $\mathcal{T}_{N}$ has a nontrivial center $\mathcal{Z}_{N}$. In particular, for any $N$, if $\alpha^{0}$ is a vector such that $\alpha_{i j}^{0}=1$, then $\operatorname{M}\left(\alpha^{0}\right)$ belongs to $\mathcal{Z}_{N}$.

Theorem 2.8. Let $\chi(N)$ stand for $N / 2$ if $N$ is even and for $(N-1) / 2$ if $N$ is odd.

(1) If $\mathrm{M}(\alpha) \in \mathcal{Z}_{N}$, then its weights have the following symmetries:

$$
\alpha_{i j}=\alpha_{j-i, N+1-i}=\alpha_{j-i, j}=\alpha_{N+1-j, N+1-i}=\alpha_{i, N+1+i-j},
$$

and $\mathrm{M}(\alpha)$ is invariant under the action of $\rho$ and $\mu_{k}$ defined in (10) and (11).

(2) If $\mathrm{M}(\alpha), \operatorname{M}\left(\alpha^{\prime}\right) \in \mathcal{Z}_{N}$, and $\alpha_{1 j}=\alpha_{1 j}^{\prime}$ for $j=2, \ldots, \chi(N)+1$, then $\alpha=\alpha^{\prime}$ and hence $\mathrm{M}(\alpha)=\mathrm{M}\left(\alpha^{\prime}\right)$. 
(3) Given $N$ and an arbitrary integer sequence $\beta_{1}, \ldots, \beta_{\chi(N)}$, there exist $m \in \mathbb{Z}_{\geq 0}$ and a vector $\alpha \in \mathbb{Z}_{\geq 0}^{N(N-1) / 2}$ such that $\mathrm{M}(\alpha) \in \mathcal{Z}_{N}$ and $\alpha_{1 j}=\beta_{j-1}+m$ for $j=$ $2, \ldots, \chi(N)+1$.

In other words, each monomial central element $\mathrm{M}(\alpha) \in \mathcal{Z}_{N}$ is uniquely determined by its weights assigned to the vertices comprising a half of a boundary side of the quiver $Q_{N}$. It follows also that the dimension of $\mathcal{Z}_{N}$ is $\chi(N)$.

For instance, for $N=3$, we have $\chi(3)=1$ and $\mathrm{M}(\alpha) \in \mathcal{Z}_{3}$ iff $\alpha_{13}=\alpha_{23}=\alpha_{12}$. Thus, $\mathcal{Z}_{3}$ is generated by $Z_{12} Z_{13} Z_{23}$. For $N=4$, we have $\chi(4)=2$ and $\mathrm{M}(\alpha) \in \mathcal{Z}_{4}$ iff $\alpha_{14}=\alpha_{34}=\alpha_{12}$ and $\alpha_{23}=\alpha_{24}=\alpha_{13}$. Thus, $\mathcal{Z}_{4}$ is generated by $Z_{12} Z_{14} Z_{34}$ and $Z_{13} Z_{23} Z_{24}$.

For $N=5$, we have $\chi(5)=2$ and $\mathrm{M}(\alpha) \in \mathcal{Z}_{5}$ iff $\alpha_{15}=\alpha_{45}=\alpha_{12}, \alpha_{14}=\alpha_{25}=\alpha_{35}=\alpha_{23}=$ $\alpha_{34}=\alpha_{13}$, and $\alpha_{24}=2 \alpha_{13}-\alpha_{12}$. The last relation requires that $2 \alpha_{13} \geq \alpha_{12}$.

Remark 2.9. The last part of Theorem 2.8 shows that the $\chi(N)$ weights that define a central monomial can be taken almost arbitrary (possibly, up to a total shift by an integer $m$ ). A direct inspection up to $N=8$ suggests that in order to have $m=0$, it suffices to take a nondecreasing integer sequence, $0 \leq \beta_{1} \leq \cdots \leq \beta_{\chi(N)}$.

Remark 2.10. The generators $Z_{i j}$ of $\mathcal{T}_{N}$ can be constructed as exponential functions of canonically conjugate variables $p_{k}, x_{k}$ such that $\left[x_{k}, p_{k^{\prime}}\right]=\sqrt{-1} \delta_{k k^{\prime}}$. Theorem 2.8 implies that the number of degrees of freedom of $\mathcal{T}_{N}$, that is, the minimal number of such pairs $\left(p_{k}, x_{k}\right)$ is $N(N-2) / 4$ if $N$ is even and $(N-1)^{2} / 4$ if $N$ is odd.

\subsection{A local tensor space representation for tetrahedron equation}

Let $V$ be a vector space. For $N \geq 2$, we define $S_{N}=V^{\otimes N(N-1) / 2}$. The tensor components of $S_{N}$ will be labeled in the lexicographic order by pairs of integers $(i, j)$, where $1 \leq i<j \leq$ $N$. For instance, $S_{3}=V_{12} \otimes V_{13} \otimes V_{23}$, where all $V_{i j}$ are isomorphic to $V$.

For a given $\mathrm{R} \in$ End $\left(S_{3}\right)$, we denote by $\mathrm{R}_{a b c}$ its canonical extension to a linear operator on $S_{N}$ which acts nontrivially only on the tensor components $V_{a b}, V_{a c}$, and $V_{b c}$. For example, $\mathrm{R}_{123}=\mathrm{R} \otimes i d \otimes i d \otimes i d$ if $N=4$.

Definition 2.11. Let $\mathrm{R} \in$ End $\left(S_{3}\right)$ be invertible and let its action canonically extended to $S_{4}$ satisfy the tetrahedron equation,

$$
\mathrm{R}_{123} \mathrm{R}_{124} \mathrm{R}_{134} \mathrm{R}_{234}=\mathrm{R}_{234} \mathrm{R}_{134} \mathrm{R}_{124} \mathrm{R}_{123} .
$$


A local tensor space representation of $\mathcal{B}(3, N)$ is a homomorphism $\mathcal{B}(3, N) \rightarrow \operatorname{End}\left(S_{N}\right)$ sending $R_{a b c}$ to $\mathrm{R}_{a b c}$.

Note that the commutativity relations (19) hold by construction. Indeed, if $\mathbf{R}_{a b c}$ and $R_{a^{\prime} b^{\prime} c^{\prime}}$ have no common pair of indices, then they act nontrivially on different tensor components of $S_{N}$ and hence they commute.

Remark 2.12. An equation formally identical to (38) was considered in [6]. There, however, it was treated as an operator equation on $V^{\otimes 4}$ with the identification $\mathrm{R}_{123}=\mathrm{R} \otimes i d$ etc. We treat (38) as an equation on $V^{\otimes 6}$, which is the standard Zamolodchikov's tetrahedron equation [15] but with a nonstandard (double index) labeling of the tensor components.

Let $V$ be the vector space of formal series in $x, x^{-1}$. Then $S_{N}$ is the vector space of formal series in $x_{i j}, x_{i j}^{-1}$, where $1 \leq i<j \leq N$.

Define operators $\mathrm{x}_{i j}, \mathrm{y}_{i j} \in \operatorname{End}\left(S_{N}\right), 1 \leq i<j \leq N$ such that

$$
\begin{aligned}
& \left(\mathrm{x}_{i j} f\right)\left(x_{12}, \ldots, x_{i j}, \ldots\right)=x_{i j} f\left(x_{12}, \ldots, x_{i j}, \ldots\right), \\
& \left(y_{i j} f\right)\left(x_{12}, \ldots, x_{i j}, \ldots\right)=f\left(x_{12}, \ldots, q x_{i j}, \ldots\right)
\end{aligned}
$$

for any $f \in S_{N}$. These operators comprise $\left(\begin{array}{c}N \\ 2\end{array}\right) q$-commuting pairs,

$$
\mathrm{x}_{i j} \mathrm{x}_{i^{\prime} j^{\prime}}=\mathrm{x}_{i^{\prime} j^{\prime}} \mathrm{x}_{i j}, \quad \mathrm{y}_{i j} \mathrm{x}_{i^{\prime} j^{\prime}}=q^{\delta_{i i^{\prime}} \delta_{j j^{\prime}}} \mathrm{x}_{i^{\prime} j^{\prime}} \mathrm{y}_{i j}, \quad \mathrm{y}_{i j} \mathrm{y}_{i^{\prime} j^{\prime}}=\mathrm{y}_{i^{\prime} j^{\prime}} \mathrm{y}_{i j}
$$

Consider $\mathrm{F} \in$ End $\left(S_{3}\right)$ whose action on monomials is given by

$$
\mathrm{F}: x_{12}^{k} x_{13}^{l} x_{23}^{m} \rightarrow x_{12}^{k} x_{13}^{m+k} x_{23}^{l-k}
$$

or, equivalently, $(\mathrm{F} f)\left(x_{12}, x_{13}, x_{23}\right)=f\left(\frac{x_{12} x_{13}}{x_{23}}, x_{23}, x_{13}\right)$. It is easy to check that

$$
\begin{aligned}
& \mathrm{Fx}_{12}=\mathrm{x}_{12} \mathrm{x}_{13} \mathrm{x}_{23}^{-1} \mathrm{~F}, \quad \mathrm{Fx}_{13}=\mathrm{x}_{23} \mathrm{~F}, \quad \mathrm{Fx}_{23}=\mathrm{x}_{13} \mathrm{~F}, \\
& \mathrm{Fy}_{12}=\mathrm{y}_{12} \mathrm{~F}, \quad F \mathrm{y}_{13}=\mathrm{y}_{12} \mathrm{y}_{23} \mathrm{~F}, \quad F \mathrm{Fy}_{23}=\mathrm{y}_{12}^{-1} \mathrm{y}_{13} \mathrm{~F} .
\end{aligned}
$$

Clearly, $\mathrm{F}$ is invertible and $\mathrm{F}^{2}=i d$. 
Let $\mathrm{R} \in \operatorname{End}\left(S_{3}\right)$ be the following operator:

$$
\mathrm{R}=\mathrm{F} \cdot\left\langle q^{1+\gamma} \mathbf{x}_{12} \mathbf{x}_{23}^{-1} \mathrm{y}_{12}^{\gamma} \mathrm{y}_{13}^{-\gamma} \mathrm{y}_{23}^{-1-\gamma}\right\rangle_{q}
$$

where $\gamma \in \mathbb{Z}$. A reader familiar with the Yang-Baxter equation will see in (44) an analogy with the standard ansatz for $R$-matrix, $\mathrm{R}=\mathrm{P} \cdot \check{\mathrm{R}}$, where $\mathrm{P}$ is the permutation, $\mathrm{P}^{2}=i d$.

Lemma 2.13. Let $F_{a b c}$ and $R_{a b c}$ stand for the canonical extensions of $F$ defined by (41) and $\mathrm{R}$ defined by (44) to operators on $S_{N}$. The homomorphisms $\theta, \phi_{\gamma}: \mathcal{B}(3, N) \rightarrow$ End $\left(S_{N}\right)$ such that $\theta\left(R_{a b c}\right)=\mathrm{F}_{a b c}$ and $\phi_{\gamma}\left(R_{a b c}\right)=\mathrm{R}_{a b c}$ are local tensor space representations of $\mathcal{B}(3, N) . \square$

Remark 2.14. The above lemma generalizes two previously known solutions of the tetrahedron equation. Namely, the case corresponding to $\gamma=0$ was found in [13], and the case corresponding to $\gamma=-1$ was considered in [9]. Our proof follows closely that given in [9].

Lemma 2.15. Let $\mathrm{x}_{a b}, \mathrm{y}_{a b}, 1 \leq a<b \leq N+1$ act on $S_{N+1}$ as defined in (39) and let $\mathrm{y}_{a b} \equiv 1$ if $a=b$. Then, for all $N \geq 2$, the linear homomorphism $\tau: \mathcal{T}_{N} \rightarrow \operatorname{End}\left(S_{N+1}\right)$ such that

$$
\tau\left(\mathrm{Z}_{a b}\right)=q \frac{\mathrm{x}_{a b}}{\mathrm{x}_{a+1, b+1}} \frac{\mathrm{y}_{a+1, b}}{\mathrm{y}_{a+1, b+1}} \text { for } 1 \leq a<b \leq N
$$

is a faithful representation of $\mathcal{T}_{N}$.

\subsection{Proof of Theorem 1.7}

One of the three symmetries of $\mathrm{T}_{N}$ presented in (16) can be established by reordering commuting factors. Namely, applying $\mu_{2}$ to relations (34) and (35) and using Lemma A.2 (see Appendix A.4), we obtain the following statement.

Lemma 2.16. For all $N \geq 2$, we have

$$
\mu_{2}\left(\mathrm{~T}_{N}\right)=\mathrm{T}_{N}
$$

Clearly, in order to establish an analogous equality involving $\mu_{1}$ or $\mu_{3}$, we will have to use the 4-4 relation (8). To do it for $\mu_{1}$, we establish a connection between the element $\mathrm{T}_{N}$ evaluated in the representation $\tau$ and words from $\mathcal{B}(3, N+1)$ evaluated in the representation $\phi_{\gamma}$ (we will take $\gamma=0$ for simplicity). 
Lemma 2.17. For all $N \geq 2$, we have the equalities

$$
\begin{aligned}
& \phi_{0}\left(W^{\prime}(3, N+1)\right)=\theta\left(W^{\prime}(3, N+1)\right) \tau\left(\mathrm{T}_{N}\right), \\
& \phi_{0}\left(\bar{W}^{\prime}(3, N+1)\right)=\theta\left(\bar{W}^{\prime}(3, N+1)\right) \tau\left(\mu_{1}\left(\mathrm{~T}_{N}\right)\right) .
\end{aligned}
$$

Invoking Theorem 2.4, we infer that $\tau\left(\mathrm{T}_{N}\right)=\tau\left(\mu_{1}\left(\mathrm{~T}_{N}\right)\right)$, which, by virtue of Lemma 2.15, implies that

$$
\mu_{1}\left(\mathrm{~T}_{N}\right)=\mathrm{T}_{N} .
$$

Now, combining (46) with (49) and using (12), we conclude that $\rho\left(\mathrm{T}_{N}\right)=\left(\mu_{1} \circ \mu_{2}\right)$ $\left(\mathrm{T}_{N}\right)=\mathrm{T}_{N}$. And finally, using (12) again, we obtain $\mu_{3}\left(\mathrm{~T}_{N}\right)=\left(\mu_{2} \circ \rho\right)\left(\mathrm{T}_{N}\right)=\mathrm{T}_{N}$.

Thus, we have obtained all relations given in Theorem 1.7.

\section{Funding}

This work was supported in part by the Swiss National Science Foundation grants 200020-140985, 200020-141329, and PDAMP2-137151, and by the Russian Fund for Basic Research grants 11-0100570 and 13-01-12405-ofi-m. The authors thank Anton Alekseev, Ludwig Faddeev, Sergey Fomin, and Rinat Kashaev for helpful conversations.

\section{Appendix}

\section{A.1 Proofs of propositions of Section 2.1}

Proof of Lemma 2.3. Let $\lfloor W\rfloor_{N+1} \in \mathcal{B}(n, N+1)$ denote the word which is obtained from a word $W \in \mathcal{B}(n-1, N)$ by the replacement $R_{a_{1}, \ldots, a_{n-1}} \rightarrow R_{a_{1}, \ldots, a_{n-1}, N+1}$ applied to all factors. A key step in proving Lemma 2.3 is to observe that the following recursive relation holds:

$$
W(n, N)\lfloor W(n-1, N)\rfloor_{N+1}=W(n, N+1) .
$$

For instance,

$$
W(2,3)\lfloor W(1,3)\rfloor_{4}=R_{12} R_{13} R_{23}\left\lfloor R_{1} R_{2} R_{3}\right\rfloor_{4}=R_{12} R_{13} R_{14} R_{23} R_{24} R_{34}=W(2,4) .
$$

For $n=2$, Equation (A.1) is almost trivial since $\lfloor W(1, N)\rfloor_{N+1}=R_{1, N+1} \cdots R_{N, N+1}$ and for each factor $R_{a, N+1}, a \neq N$ the rightmost factor in $W(2, N)$ with which it does not commute is $R_{a, N}$. Therefore, moving all factors from $\lfloor W(1, N)\rfloor_{N+1}$ to the left until they meet their noncommuting counterparts, we obtain the lexicographically ordered 
word $W(2, N+1)$. Note that only the last two factors in $\lfloor W(1, N)\rfloor_{N+1}$ have the same noncommuting counterpart, namely, $R_{N-1, N}$.

The proof for $n \geq 3$ is similar. Each factor $R_{a_{1}, \ldots, a_{n-1}, N+1}$ in $\lfloor W(n-1, N)\rfloor_{N+1}$ belongs to a cluster, that is, a product of consecutive lexicographically ordered factors. A cluster has length 1 if $a_{n-1}<N-1$. If $a_{n-1} \geq N-1$, then the length of a cluster is $k+1$, where $k=\max \left\{m: a_{n-m} \geq N-m\right\}$. If $R_{a_{1}, \ldots, a_{n-k-1}, N-k, \ldots, N-1, N+1}$ is the leftmost factor of a cluster of length $k+1$, then the rightmost noncommuting counterpart in $W(n, N)$ for all factors of the cluster is $R_{a_{1}, \ldots, a_{n-k-1}, N-k, \ldots, N-1, N}$. Therefore, moving each cluster from $\lfloor W(n-1, N)\rfloor_{N+1}$ to the left until it meets its counterpart, we achieve the lexicographic order of all factors, that is, we obtain the word $W(n, N+1)$.

The recursive relation (A.1) implies that

$$
W(n, N)=\overrightarrow{\prod_{n \leq k \leq N}}\lfloor W(n-1, k-1)\rfloor_{k},
$$

which in turn leads to the expression (24) for $W^{\prime}(n, N)$.

To prove Theorem 2.4, we will need also the following statement.

Lemma A.1. Let $\omega$ be an involutive automorphism of $\mathcal{B}(n, N)$ such that

$$
\omega\left(R_{a_{1}, \ldots, a_{n}}\right)=R_{N+1-a_{n}, \ldots, N+1-a_{1}}
$$

for all generators of $\mathcal{B}(n, N)$. Then, for all $N \geq n$, the equalities

$$
\omega(W(n, N))=\eta\left(W^{\prime}(n, N)\right), \quad \omega\left(W^{\prime}(n, N)\right)=\eta(W(n, N))
$$

hold in the strong sense, that is, their r.h.s. coincide with their l.h.s. without a permutation of commuting factors.

Proof. Define $\bar{W}^{\prime}(n, N)=\eta\left(W^{\prime}(n, N)\right)$. Applying the anti-automorphism $\eta$ to (24) and (A.1), we obtain

$$
\begin{array}{r}
\bar{W}^{\prime}(n, N)=\prod_{1 \leq b_{1}<\cdots<b_{n} \leq N}{ }_{\prod_{b_{1}, \ldots, b_{n}}}^{\prime} \\
\bar{W}^{\prime}(n, N+1)=\left\lfloor\bar{W}^{\prime}(n-1, N)\right\rfloor_{N+1} \bar{W}^{\prime}(n, N)
\end{array}
$$


Applying to (A.5) the automorphism $\omega$, and relabeling the indices by $a_{k}=N+1-b_{n+1-k}$, we recover the r.h.s. of formula (23). Hence we infer that $\omega\left(\eta\left(W^{\prime}(n, N)\right)\right)=W(n, N)$, which proves the first equality in (A.4). The second equality follows then immediately since $\omega$ and $\eta$ mutually commute and both are involutive.

Proof of Theorem 2.4. For $n=1$, the statement of the theorem is obvious since all $R^{\prime} \mathrm{s}$ commute. For $n=2$, the statement can be proved by induction on $N$. The base, for $N=3$, is simply the relation (20). Assume that the equality $W(2, N)=\bar{W}(2, N)$ has been already established for some $N \geq 3$. Then, taking into account the recursive structure of (A.1) and (A.6), we have to prove that

$$
\left\lfloor\prod_{1 \leq a \leq N} R_{a}\right\rfloor_{N+1}=(W(2, N))^{-1}\left\lfloor\prod_{1 \leq a \leq N} R_{a}\right\rfloor_{N+1} W(2, N)
$$

Observe that the Yang-Baxter equation (21) can be rewritten as the following "almost commutativity" relation for two $R^{\prime}$ :

$$
\left\lfloor R_{a} R_{b}\right\rfloor_{N+1}=R_{a b}^{-1}\left\lfloor R_{b} R_{a}\right\rfloor_{N+1} R_{a b}
$$

Using this relation, we can move $R_{1, N+1}$ in the product $\vec{\prod} R_{a, N+1}$ to the right, then move $R_{2, N+1}$, and so on until we obtain the reverse-ordered product $\overleftarrow{\prod} R_{a, N+1}$. Note that all the extra "twisting" factors $R_{a b}^{ \pm 1}$ arising in this process commute with any $R_{C, N+1}, c \neq$ $a, b$. Therefore, at each step these twisting factors can be moved outside of the product $\lfloor\cdots\rfloor_{N+1}$. It is easy to see that these factors combine into $(W(2, N))^{ \pm 1}$. Thus, we have established the inductive step (A.7) and hence the Theorem is proved for $n=2$.

For $n=3$, the theorem can be proved along the same lines. The base, for $N=4$, is the relation (20). Assuming that $W(3, N)=\bar{W}(3, N)$ has been already established for some $N \geq 4$ and taking into account (A.1) and (A.6), we have to prove the inductive step:

$$
\lfloor W(2, N)\rfloor_{N+1}=(W(3, N))^{-1}\lfloor\bar{W}(2, N)\rfloor_{N+1} W(3, N) .
$$

Observe that the tetrahedron Equation (22) can be rewritten as the following "almost Yang-Baxter" relation for three $R^{\prime}$ :

$$
\left\lfloor R_{a b} R_{a c} R_{b c}\right\rfloor_{N+1}=R_{a b c}^{-1}\left\lfloor R_{b c} R_{a c} R_{a b}\right\rfloor_{N+1} R_{a b c},
$$

where $a<b<c$. Note that $R_{a b c}$ commutes with any factor in $\lfloor W(2, N)\rfloor_{N+1}$ except those on the 1.h.s. of (A.10). Therefore, the factors in $\lfloor W(2, N)\rfloor_{N+1}$ can be reordered exactly in the 
same way as the factors in $W(2, N)$ and the extra twisting factors $R_{a b c}^{ \pm 1}$ arising at each step can be moved outside of the product $[\cdots\rfloor_{N+1}$. It is easy to see that these factors combine into $(W(3, N))^{ \pm 1}$. Since we already know that $W(2, N)=\bar{W}(2, N)$, it follows that the inductive step (A.9) holds and so the theorem is proved for $n=3$.

It is now clear that the proof continues by the double induction on $n$ and $N$. For a given $n$, relation (20) can be rewritten as "almost" the relation for $n-1$. Using it, we can reorder the factors in $\lfloor W(n-1, N)\rfloor_{N+1}$ exactly in the same way as the factors in $W(n-1, N)$. At each step the extra twisting factors can be moved outside of the product $\lfloor\cdots\rfloor_{N+1}$ due to relation (19). Therefore, the same line of arguments as for $n=2,3$ proves the theorem for an arbitrary $n$.

Proof of Lemma 2.7. (1) The first equality in (33) is obvious from Definition 1.5 and Equation (31). In order to prove the second equality in (33), we consider the following elements of $\mathcal{T}_{N}$ :

$$
\mathrm{T}_{N, b}=\prod_{1 \leq a<k \leq b} \mathbb{R}_{a k}, \quad 2 \leq b \leq N .
$$

We observe that

$$
\mathrm{T}_{N, b}=\mathrm{T}_{N, b-1} \prod_{1 \leq a<b} \mathbb{R}_{a b}
$$

Indeed, it follows from the definition (31) that the rightmost factor in the lexicographically ordered product $T_{N, b}$ which does not commute with $\mathbb{R}_{a b}$ is $\mathbb{R}_{a, b-1}$. Repeating the argument used in the proof of formula (A.1) for $n=2$, we conclude that (A.12) holds. And it is easy to see that (A.12) along with $\mathrm{T}_{N}=\mathrm{T}_{N, N}$ implies that

$$
\mathrm{T}_{N}=\mathrm{T}_{N, N-1} \overrightarrow{\prod_{1 \leq a<N}} \mathbb{R}_{a N}=\cdots=\vec{\prod}_{1 \leq a<b \leq N}^{\prime} \mathbb{R}_{a b}=\left(W^{\prime}(2, N)\right)^{\sharp} .
$$

(2) The first equality in (34) is obvious from Definition 1.5. The second equality in (34) is equivalent to the statement that

$$
\mathrm{T}_{N}=\mathrm{T}_{N-1} \mathrm{~W}^{\prime}(2, N),
$$

where $\mathrm{W}^{\prime}(2, N)$ is given by (29) with each $R_{a b}$ replaced with $\mathbb{R}_{a, b, N+1}$. Using relations (A.1) and (A.13), we can rewrite the r.h.s. of (A.14) as follows:

$$
\tilde{\mathbf{W}}^{\prime}(2, N-1) \mathrm{W}^{\prime}(2, N-1)\left(\prod_{1 \leq e<N} \mathbb{R}_{e N}\right),
$$


where $\tilde{W}^{\prime}(2, N)$ is given by (29) with each $R_{a b}$ replaced with $\mathbb{R}_{a b}^{\prime}$, which is an element of $\mathcal{T}_{N-1}$ given by (31). Definition (31) implies that $\mathbb{R}_{a b}^{\prime}$ is the rightmost factor in $\tilde{W}^{\prime}(2, N-1)$ which does not commute with the factor $\mathbb{R}_{a, b, N+1}$ in $\mathrm{W}^{\prime}(2, N-1)$. Therefore, moving all factors from $W^{\prime}(2, N-1)$ to the left until they meet their noncommuting counterparts and noting that $\mathbb{R}_{a b}^{\prime} \mathbb{R}_{a, b, N+1}=\mathbb{R}_{a b}$, we conclude that (A.15) coincides with the second expression in (A.13) and hence relation (A.14) holds.

(3) To prove the last part of the lemma, we consider $\mathrm{W}^{\prime}(2, N)$ introduced in (A.14). One can check that all its factors containing $Z_{N-1, N}$ can be moved to the right preserving their order. Making then the same procedure with factors containing $Z_{N-2, N}, Z_{N-3, N}$, etc., we conclude that $\mathrm{W}^{\prime}(2, N)$ is given by the same expression in (29) where each $R_{a b}$ is replaced with $\mathbb{R}_{a, N+1+a-b, N+1}$. With such a form of $\mathrm{W}^{\prime}(2, N)$ formula (A.14) leads immediately to the second equality in (35).

Equivalence of the first and second expressions for $\mathrm{T}_{N}$ in (35) is verified as follows:

$$
(W(3, N))^{\star \star}=\mu_{2}\left(\left(W^{\prime}(3, N)\right)^{\star}\right)=\mu_{2}\left((W(3, N))^{\star}\right)=\left(W^{\prime}(3, N)\right)^{\star \star} .
$$

The middle equality is due to the second part of the lemma. In the first and last equalities, we used Lemma A.2, which is proved below in Section A.4.

\section{A.2 Proof of Theorem 2.8}

Recall that $\alpha \in \mathbb{Z}_{\geq 0}^{N(N-1) / 2}$ is a vector comprising the weights $\alpha_{i j}$ assigned to the vertices of $Q_{N}$ ordered lexicographically. By (36), each $\alpha$ determines a monomial $\mathrm{M}(\alpha) \in \mathcal{T}_{N}$. Relations (9) imply that $\mathrm{M}(\alpha)$ commutes with all generators of $\mathcal{T}_{N}$ iff $B \alpha=0$. Thus, we have to study the kernel of the incidence matrix $B$.

(1) Let $\mathcal{U}$ be the symmetry transformation of the vertices of $Q_{N}$ such that $\mathcal{U}(i, j)=$ ( $j-i, N+1-i$ ), and $U$ be the matrix of the corresponding orthogonal transformation

of the basis in $\mathbb{Z}_{\geq 0}^{N(N-1) / 2}$. Applying $\rho$ to (9), we infer that $B$ commutes with $U$. Taking into account that $U$ is orthogonal and $B$ is skew-symmetric, we conclude that if $\alpha \in \operatorname{Ker} B$, then $\alpha$ is an eigenvector of $U$. However, $U^{3}=1$ and so the only real eigenvalue of $U$ is 1 . Thus, $\mathrm{M}(\alpha) \in \mathcal{Z}_{N}$ implies that $U \alpha=\alpha$. Hence

$$
\alpha_{\mathcal{U}(i, j)}=\alpha_{i j},
$$

which is the first symmetry in (37). Applying $\rho$ to a central monomial $\mathrm{M}(\alpha)$ and taking this symmetry of its weights into account, we infer that $\rho(\mathrm{M}(\alpha))=q^{\varepsilon(\alpha)} \mathrm{M}(\alpha)$, where the 
multiplicative constant appears due to reordering of the generators. However, the property $\rho \circ \rho \circ \rho=i d$ implies that $q^{\varepsilon(\alpha)}=1$ and thus $\rho(\mathrm{M}(\alpha))=\mathrm{M}(\alpha)$.

Let $\mu$ be any of the anti-homomorphisms in (11), $\mathcal{K}$ be the corresponding symmetry transformation of the vertices of $Q_{N}$ (e.g., $\mathcal{K}(i, j)=(j-i, j)$ for $\left.\mu_{1}\right)$, and $K$ be the matrix of the corresponding orthogonal transformation of the basis in $\mathbb{Z}_{\geq 0}^{N(N-1) / 2}$. Applying $\mu$ to (9), we infer that $K B K=-B$. Hence $B^{2}$ commutes with $K$. Taking into account that both these matrices are real symmetric and $K^{2}=1$, we conclude that if $\alpha \in \operatorname{Ker} B$, then $K \alpha= \pm \alpha$. But $\alpha_{12}=\alpha_{1 N}=\alpha_{N-1, N}$ owing to (A.17). Therefore, $K \alpha=\alpha$. Hence

$$
\alpha_{\mathcal{K}(i, j)}=\alpha_{i j},
$$

which yields the remaining symmetries in (37). Applying $\mu$ to a central monomial $M(\alpha)$ and taking these symmetries into account, we obtain that $\mu(\mathrm{M}(\alpha))=q^{\varepsilon^{\prime}(\alpha)} \mathrm{M}(\alpha)$. The property $\mu \circ \mu=i d$ implies that $q^{\varepsilon^{\prime}(\alpha)}=1$ and thus $\mu(\mathrm{M}(\alpha))=\mathrm{M}(\alpha)$.

(2) Consider monomials $\mathrm{M}(\alpha)$ and $\mathrm{M}\left(\alpha^{\prime}\right)$ such that $\alpha_{1 j}=\alpha_{1 j}^{\prime}$ for $j=2, \ldots, N$. Then, in view of (A.17), all components of vector $\alpha^{\prime \prime}=\alpha-\alpha^{\prime}$ corresponding to boundary vertices of $Q_{N}$ vanish. Suppose that $\mathrm{M}(\alpha)$ and $\mathrm{M}\left(\alpha^{\prime}\right)$ are central. Then $\mathrm{M}\left(\alpha^{\prime \prime}\right)$ commutes with all generators of $\mathcal{T}_{N}$. In particular, the condition that $\mathrm{M}\left(\alpha^{\prime \prime}\right)$ commutes with all $Z_{1 j}$ is equivalent to a system of equations: $\alpha_{2, j+1}^{\prime \prime}=\alpha_{2, j}^{\prime \prime}$. However, we already have $\alpha_{23}^{\prime \prime}=0$. Therefore, we conclude that $\alpha_{2 j}^{\prime \prime}=0$ for $j=3, \ldots, N$. This implies, in view of (A.17), that all components of $\alpha^{\prime \prime}$ corresponding to next to boundary vertices of $Q_{N}$ also vanish. Continuing this consideration similarly for $Z_{2 j}, Z_{3 j}$, etc., we conclude that $\alpha^{\prime \prime}=0$. Thus, $M\left(\alpha^{\prime}\right)=\mathrm{M}(\alpha)$, that is, two central monomials coincide iff they have coinciding weights at one boundary of $Q_{N}$. Owing to the symmetry (A.18), the latter condition is equivalent to a weaker condition: $\alpha_{1 j}^{\prime}=\alpha_{1 j}$ for $j=2, \ldots, \chi(N)+1$.

(3) Let us show that, given arbitrary integers $\beta_{1}, \ldots, \beta_{\chi(N)}$, there exists a unique integer vector $\tilde{\alpha}$ such that $\tilde{\alpha}_{1 j}=\beta_{j-1}$ for $j=2, \ldots, \chi(N)+1$ and $\mathrm{M}(\tilde{\alpha})$ given by (36) (where some $\tilde{\alpha}_{i j}$ can be negative) commutes with all generators $Z_{i j}$. Let $\partial Q_{N}$ stand for the set of all boundary vertices of $Q_{N}$. Forgetting about the edges, we have $Q_{N}=\partial Q_{N} \cup$ $\partial Q_{N-3} \cup \cdots$.

First, given the weights $\tilde{\alpha}_{1 j}$ for $j=2, \ldots, \chi(N)+1$, we extend them to weights at other vertices of $\partial Q_{N}$ by the symmetries (A.17) and (A.18). Now, the requirement that $\mathrm{M}(\tilde{\alpha})$ commutes with all generators assigned to $\partial Q_{N}$ fixes uniquely all weights at $\partial Q_{N-3}$. Indeed, $\mathrm{M}(\tilde{\alpha})$ commutes with $Z_{1 j}$ if $\tilde{\alpha}_{2, j+1}-\tilde{\alpha}_{2 j}=\tilde{\alpha}_{1, j+1}-\tilde{\alpha}_{1, j-1}$. Taking into account that $\tilde{\alpha}_{23}=\tilde{\alpha}_{13}$, this set of equations determines all $\tilde{\alpha}_{2 j}$ uniquely. Moreover, the symmetry (A.18) for $\tilde{\alpha}_{1 j}$ induces the same symmetry for $\tilde{\alpha}_{2 j}$. Finally, $\tilde{\alpha}_{2 j}$ can be extended to weights at other vertices of $\partial Q_{N-3}$ by the symmetry (A.17). 
Similarly, given weights at $\partial Q_{k+3}$ and $\partial Q_{k}$, the requirement that $\mathrm{M}(\tilde{\alpha})$ commutes with all generators assigned to $\partial Q_{k}$ fixes uniquely all weights at $\partial Q_{k-3}$. Indeed, $\operatorname{M}(\tilde{\alpha})$ commutes with all $Z_{i j}$ for a given $i$ if $\tilde{\alpha}_{i+1, j+1}-\tilde{\alpha}_{i+1, j}=\tilde{\alpha}_{i, j+1}-\tilde{\alpha}_{i, j-1}+\tilde{\alpha}_{i-1, j-1}-\tilde{\alpha}_{i-1, j}$. These equations are resolved uniquely since we know the r.h.s. and several first values of $\tilde{\alpha}_{i+1, j}$ owing to the symmetry (A.17).

Thus, the vector $\tilde{\alpha}$ is recovered uniquely from its first $\chi(N)$ components. However, it can happen that some $\tilde{\alpha}_{i j}$ are negative. In this case, we take another vector, $\alpha=\tilde{\alpha}+m \alpha^{0}$, where $\alpha^{0}$ is a vector such that $\alpha_{i j}^{0}=1$, and $m$ is a sufficiently large positive number to ensure the positivity of all $\alpha_{i j}$. Then we have $\mathrm{M}(\alpha)=q^{\varepsilon} \cdot \mathrm{M}(\tilde{\alpha})\left(\mathrm{M}\left(\alpha^{0}\right)\right)^{m}$. It remains to observe that $\mathrm{M}\left(\alpha^{0}\right) \in \mathcal{Z}_{N}$ and hence $\mathrm{M}(\alpha) \in \mathcal{Z}_{N}$.

\section{A.3 Proofs of propositions of Section 2.3}

Proof of Lemma 2.13. The statement of the Lemma for $F$ follows from the fact that the action of $F_{123} F_{124} F_{134} F_{234}$ and $F_{234} F_{134} F_{124} F_{123}$ on the monomial $x_{12}^{a} x_{13}^{b} x_{23}^{c} x_{14}^{d} x_{24}^{e} x_{34}^{f}$ yields the same result.

Note that $R$ given by (44) is invertible. If the argument of the quantum exponential in (44) is denoted by $\mathrm{X}$, then we have $\mathrm{R}^{-1}=\langle\mathrm{X}\rangle_{q}^{-1} \mathrm{~F}$, where $\langle x\rangle_{q}^{-1}$ is the following formal series:

$$
\langle x\rangle_{q}^{-1}=\sum_{n \geq 0} \frac{q^{n(n-1) / 2} x^{n}}{(1-q) \cdots\left(1-q^{n}\right)} .
$$

In order to check that $\mathrm{R}$ satisfies the tetrahedron equation, one has to substitute $\mathrm{R}_{a b c}$ into (38), move all F's to the left, and cancel the products of F's on the both sides of the equation by invoking the first part of the lemma. Then one is left with the equality

$$
\langle\mathrm{X}\rangle_{q}\langle\mathrm{XY}\rangle_{q}\langle\mathrm{Z}\rangle_{q}\langle\mathrm{Y}\rangle_{q}=\langle\mathrm{Z}\rangle_{q}\langle\mathrm{ZX}\rangle_{q}\langle\mathrm{Y}\rangle_{q}\langle\mathrm{X}\rangle_{q}
$$

where

$$
\begin{aligned}
& \mathrm{X}=q^{1+\gamma} \mathrm{x}_{12} \mathrm{x}_{23}^{-1} \mathrm{y}_{12}^{\gamma} \mathrm{y}_{13}^{-\gamma} \mathrm{y}_{23}^{-1-\gamma}, \quad \mathrm{Y}=q^{1+\gamma} \mathrm{x}_{23} \mathrm{x}_{34}^{-1} \mathrm{y}_{23}^{\gamma} \mathrm{y}_{24}^{-\gamma} \mathrm{y}_{34}^{-1-\gamma}, \\
& \mathbf{Z}=q^{1+\gamma} \mathrm{x}_{13} \mathrm{x}_{24}^{-1} \mathrm{y}_{13}^{\gamma} \mathrm{y}_{14}^{-\gamma} \mathrm{y}_{23}^{1+\gamma} \mathrm{y}_{24}^{-1-\gamma} .
\end{aligned}
$$

These operators satisfy relations (5). Therefore, comparing the first equality in (8) with (A.20), we conclude that equality (A.20) holds. 
Proof of Lemma 2.15. Note that there are only six cases when $\tau\left(Z_{a b}\right)$ does not commute with $\tau\left(Z_{a^{\prime} b^{\prime}}\right)$ : (1) $a^{\prime}=a$ and $b^{\prime}=b \pm 1$; (2) $a^{\prime}-a= \pm 1$ and $b^{\prime}=b$ or $b^{\prime}=b+a^{\prime}-a$. It is easy to check that in these cases $\tau\left(Z_{a b}\right)$ and $\tau\left(Z_{a^{\prime} b^{\prime}}\right)$ satisfy the defining relations (9).

Clearly, the representation (45) is faithful for $\mathcal{T}_{2}$ since in this case the algebra has only one generator. The proof of faithfulness of this representation in other cases will use the inclusion $\mathcal{T}_{N-1} \subset \mathcal{T}_{N}$ (cf. Remark 1.3). Let $N$ be the minimal positive number such that the representation $\tau$ is not faithful for $\mathcal{T}_{N}$. Then there exists a polynomial $H$ in $\left(\begin{array}{c}N \\ 2\end{array}\right)$ variables such that $\mathrm{H} \equiv H\left(\tau\left(\mathrm{Z}_{12}\right), \ldots, \tau\left(\mathrm{Z}_{N-1, N}\right)\right)=0$.

Without a loss of generality, we can assume that $\mathrm{H}$ has the form

$$
\mathrm{H}=\sum_{k_{1}, \ldots, k_{N-1} \geq 0} B_{k_{1} \cdots k_{N-1}} \tau\left(\mathbf{Z}_{1 N}^{k_{1}} \cdots \mathbf{Z}_{N-1, N}^{k_{N-1}}\right) \mathrm{H}_{k_{1} \cdots k_{N-1}}
$$

where $B_{k_{1} \cdots k_{N-1}} \neq 0$ at least for one set $\left\{k_{1}, \ldots, k_{N-1}\right\}$ such that $k_{1}+\cdots+k_{N-1}>0$. Here $\mathrm{H}_{k_{1} \cdots k_{N-1}}$ stand for polynomials in the generators of $\mathcal{T}_{N-1}$ evaluated in the representation (45).

Acting by $\mathrm{H}$ on $f \in S_{N} \subset S_{N+1}$, we obtain

$$
\mathrm{H} \cdot f=\sum_{k_{1} \cdots k_{N-1} \geq 0} q^{\varepsilon\left(k_{1}, \ldots, k_{N-1}\right)} B_{k_{1} \cdots k_{N-1}}\left(\prod_{a=1}^{N-1}\left(\frac{x_{a N}}{x_{a+1, N+1}}\right)^{k_{a}}\right)\left(\tilde{\mathrm{H}}_{k_{1} \cdots k_{N-1}} \cdot f\right),
$$

where $\tilde{\mathrm{H}}_{k_{1} \cdots k_{N-1}}=\mathrm{Y}_{N} \mathrm{H}_{k_{1} \cdots k_{N-1}}$ with $\mathrm{Y}_{N}=\mathrm{y}_{2 N} \cdots \mathrm{y}_{N-1, N}$. Note that all terms in the sum in (A.23) are linearly independent monomials in $x_{2, N+1}^{-1}, \ldots, x_{N, N+1}^{-1}$. Hence, $\mathrm{H}=0$ implies that $\tilde{\mathrm{H}}_{k_{1} \cdots k_{N-1}} \cdot f=0$. Since $\mathrm{Y}_{N}$ is invertible, we conclude that $\mathrm{H}_{k_{1} \cdots k_{N-1}}$ annihilates an arbitrary $f$ from $S_{N}$ and, thus, $\mathrm{H}_{k_{1} \cdots k_{N-1}}=0$. But this contradicts the assumption that the representation $\tau$ is faithful for $\mathcal{T}_{N-1}$.

\section{A.4 Proof of propositions of Section 2.4}

Lemma A.2. For all $N \geq 3$, the equalities

$$
\mu_{2}\left((W(3, N))^{\star}\right)=\left(W^{\prime}(3, N)\right)^{\star \star}, \quad \mu_{2}\left(\left(W^{\prime}(3, N)\right)^{\star}\right)=(W(3, N))^{\star \star},
$$

hold in the strong sense, that is their r.h.s. coincide with their l.h.s. without a permutation of commuting factors. 
Proof. The first equality in (A.24) is checked with the help of Lemma A.1 as follows:

$$
\begin{aligned}
\mu_{2}\left((W(3, N))^{\star}\right) & =\mu_{2}\left(\prod_{1 \leq a<b<c \leq N}\left(R_{a b c}\right)^{\star}\right)=\prod_{1 \leq a<b<c \leq N}^{\prime} \mu_{2}\left(\left(\omega\left(R_{a b c}\right)\right)^{\star}\right) \\
& =\vec{\prod}_{1 \leq a<b<c \leq N}^{\prime} \mu_{2}\left(\mathbb{R}_{N+1-c, N+1-b, N+1-a}\right)=\vec{\prod}_{1 \leq a<b<c \leq N}^{\prime} \mathbb{R}_{a, c+a-b, c}=\left(W^{\prime}(3, N)\right)^{\star \star} .
\end{aligned}
$$

The second equality can be checked in a similar way.

Recall that the homomorphisms $\theta$ and $\phi_{\gamma}$ were introduced in Lemma 2.13. In this subsection, we will use the following notations:

$$
\mathrm{R}_{a b c}=\phi_{0}\left(R_{a b c}\right)=\mathrm{F}_{a b c} \cdot \check{\mathrm{R}}_{a b c}, \quad \mathrm{~F}_{a b c}=\theta\left(R_{a b c}\right), \quad \check{\mathrm{R}}_{a b c}=\left\langle q \mathbf{x}_{a b} \mathbf{x}_{b c}^{-1} \mathrm{y}_{b c}^{-1}\right\rangle_{q} .
$$

We will need also the following homomorphisms $\varphi, \varphi^{\prime}$ from $\mathcal{B}(3, N)$ to $\operatorname{End}\left(S_{N}\right)$ :

$$
\begin{aligned}
\varphi\left(R_{a b c}\right) & =\left\langle\frac{q \mathrm{x}_{a b}}{\mathrm{x}_{a+c-b, c}} \prod_{k=1}^{c-b} \frac{\mathrm{y}_{a+k, b+k-1}}{\mathrm{y}_{a+k, b+k}}\right\rangle_{q}, \\
\varphi^{\prime}\left(R_{a b c}\right) & =\left\langle q\left(\prod_{k=1}^{c-b} \frac{\mathrm{x}_{b-a, b+k-1}}{\mathrm{x}_{b-a+1, b+k}}\right) \frac{\mathrm{y}_{b-a+1, b}}{\mathrm{y}_{b-a+1, c}}\right\rangle_{q},
\end{aligned}
$$

where $\mathrm{y}_{a b} \equiv 1$ if $a=b$.

Recall that the word $W^{\prime}(3, N)$ is given by $(30)$, and that $\bar{W}^{\prime}(3, N)=\eta\left(W^{\prime}(3, N)\right)$ is the word with reversely ordered factors. Let us introduce

$$
\mathrm{F}_{N}=\theta\left(W^{\prime}(3, N)\right)=\theta\left(\bar{W}^{\prime}(3, N)\right)
$$

where the equality of the expressions is due to Theorem 2.4.

Lemma A.3. For all $N \geq 3$, we have the equalities

$$
\begin{aligned}
& \phi_{0}\left(W^{\prime}(3, N)\right)=\mathrm{F}_{N} \cdot \varphi\left(W^{\prime}(3, N)\right), \\
& \phi_{0}\left(\bar{W}^{\prime}(3, N)\right)=\mathrm{F}_{N} \cdot \varphi^{\prime}\left(\bar{W}^{\prime}(3, N)\right) .
\end{aligned}
$$


Proof. Equation (A.2) implies that

$$
\phi_{0}\left(W^{\prime}(3, N)\right)=\overrightarrow{\prod_{3 \leq c \leq N}} \phi_{0}\left(\left\lfloor W^{\prime}(2, c-1)\right\rfloor_{c}\right)
$$

Consider $\check{\mathrm{R}}_{a b c}$ entering the $\phi_{0}\left(\lfloor\cdots\rfloor_{c}\right)$ part. In order to establish (A.29), it suffices to prove that:

(i) $\check{\mathrm{R}}_{a b c}$ transforms into $\varphi\left(\mathrm{R}_{a b c}\right)$ when all F's from $\phi_{0}\left(\lfloor\cdots\rfloor_{c}\right)$ are moved to the left of this part;

(ii) $\varphi\left(\mathrm{R}_{a b c}\right)$ does not change when, for any $k>c$, all $\mathrm{F}^{\prime} \mathrm{s}$ from $\phi_{0}\left(\left\lfloor W^{\prime}(2, k-1)\right\rfloor_{k}\right)$ are moved through it.

Proof of (i). Consider $\check{\mathrm{R}}_{a b c}$ which is not the rightmost one (otherwise, it already has the form $\varphi(R)$ ). All $\mathrm{F}^{\prime} \mathrm{s}$ to the right of it have the form $\mathrm{F}_{a^{\prime} b^{\prime} c},\left\{a^{\prime}, b^{\prime}\right\} \neq\{a, b\}$. By (42), they do not change $\mathrm{x}_{a b}$ but act on the first index of $\mathrm{x}_{b c}$ as permutations $P_{a^{\prime} b^{\prime}}$. From the recursive structure of the word $W^{\prime}(2, c-1)=\lfloor W(1,1)\rfloor_{2} \cdots\lfloor W(1, c-2)\rfloor_{c-1}$ it follows that $x_{b c}$ has to be pulled through the following chain of permutations:

$$
P_{a+1, b} \cdots P_{b-1, b} P_{1, b+1} \cdots P_{b, b+1} \cdots P_{1, c-1} \cdots P_{C-2, c-1} .
$$

The part $P_{a+1, b} \cdots P_{b-1, b}$ here is present if $a<b-1$. It transforms $\mathrm{x}_{b c}$ into $\mathrm{x}_{a+1, c}$. The remaining permutations are grouped into $(c-1-b)$ shift operators $U_{d}=P_{d, d+1} \cdots P_{12}$, $b \leq d \leq c-2$. Each $U_{d}$ increases the first index of $\mathrm{x}_{a+1, c}$ by 1 , thus transforming it into $\mathbf{x}_{a+c-b, c}$.

Consider now $\mathrm{y}_{b c}$ entering the argument of $\check{\mathrm{R}}_{a b c}$. It is first transformed by $\mathrm{F}_{a+1, b, c}$ into $\frac{y_{a+1, c}}{y_{a+1, b}}$. Note that, by (43), all F's entering $\phi_{0}\left(\left\lfloor W^{\prime}(2, c-1)\right\rfloor_{c}\right)$ act nontrivially only on y's whose second index is $c$. From the recursive structure of the word $W^{\prime}(2, c-1)$ it follows that $\mathrm{y}_{a+1, c}$ is transformed by consecutive action of pairs $\mathrm{F}_{a+k, b+k, c} \mathrm{~F}_{a+1+k, b+k, c}, 1 \leq k \leq c-$ $b-1$. Each such pair transforms $\mathrm{y}_{a+k, c}$ into $\frac{\mathrm{y}_{a+k, b+k}}{\mathrm{y}_{a+1+k, b+k}} \mathrm{y}_{a+1+k, c}$, which yields the product of $y^{\prime} s$ in (A.26).

Proof of (ii). This part is trivial for y's since, by (43), $\mathrm{y}_{a b}$ commutes with any $\mathrm{F}_{a^{\prime} b^{\prime} k}$ if $k \neq a, b$. For $\mathrm{x}^{\prime} \mathrm{s}$ we have to consider the transformations of $\frac{\mathrm{x}_{a b}}{\mathrm{x}_{a^{\prime} c}}$, where $a^{\prime} \equiv a+c-b$. Note that $a<a^{\prime}<c$. Therefore, $\frac{\mathrm{x}_{a b}}{\mathrm{x}_{a^{\prime} c}}$ is first transformed by $\mathrm{F}_{a b k}$ into $\frac{\mathrm{x}_{a b} \mathrm{x}_{a k}}{\mathrm{x}_{a^{\prime} c} \mathrm{x}_{b k}}$. Then this expression is pulled through all F's between $F_{a b k}$ and $F_{a^{\prime} c k}$. They act only on the first indices of $\mathbf{x}_{a k}$ 
and $\mathrm{x}_{b k}$ as the following chain of permutations:

$$
P_{a+1, b} \cdots P_{b-1, b} P_{1, b+1} \cdots P_{b, b+1} \cdots P_{1, c} \cdots P_{a^{\prime}-1, c} .
$$

The part $P_{a+1, b} \cdots P_{b-1, b}$ transforms $\frac{\mathrm{x}_{a k}}{\mathrm{x}_{b k}}$ into $\frac{\mathrm{x}_{a k}}{\mathrm{x}_{a+1, k}}$. Then each of the shift operators $U_{d}$ $b \leq d \leq c-2$ increases the first indices of $\mathbf{x}_{a k}$ and $x_{a+1, k}$ by 1 , which yields $\frac{\mathrm{x}_{a^{\prime}-1, k}}{\mathrm{x}_{a^{\prime} k}}$. The last part, $P_{1, c} \cdots P_{a^{\prime}-1, c}$, transforms $\frac{x_{a^{\prime}-1, k}}{x_{a^{\prime} k}}$ into $\frac{x_{c k}}{x_{a^{\prime} k}}$. Finally, $\frac{x_{a b} x_{c k}}{x_{a^{\prime} c} x_{a^{\prime} k}}$ is transformed by $F_{a^{\prime} c k}$ into $\frac{\mathrm{x}_{a b}}{\mathrm{x}_{a^{\prime} c}}$, which then is not affected by the remaining $\mathrm{F}^{\prime} \mathrm{s}$.

Thus, we have verified (i) and (ii) and therefore proved relation (A.29).

In order to establish equation (A.30), we will prove first that

$$
\phi_{0}\left(\bar{W}^{\prime}(3, N)\right)=\varphi^{\prime \prime}\left(\bar{W}^{\prime}(3, N)\right) \cdot \mathrm{F}_{N},
$$

where $\varphi^{\prime \prime}$ is a homomorphism from $\mathcal{B}(3, N)$ to End $\left(S_{N}\right)$,

$$
\varphi^{\prime \prime}\left(R_{a b c}\right)=\left\langle\frac{q \mathbf{x}_{a b}}{\mathbf{x}_{a+c-b, c}} \prod_{k=0}^{c-b-1} \frac{\mathrm{y}_{a+k, b+k}}{\mathrm{y}_{a+k, b+k+1}}\right\rangle_{q} .
$$

Indeed, we have

$$
\mathrm{R}_{a b c}=\phi_{0}\left(R_{a b c}\right)=\hat{\mathrm{R}}_{a b c} \cdot \mathrm{F}_{a b c}, \quad \hat{\mathrm{R}}_{a b c}=\left\langle q \mathbf{x}_{a b} \mathbf{x}_{b c}^{-1} \mathrm{y}_{a b} \mathrm{y}_{a c}^{-1}\right\rangle_{q}
$$

Note that the x's arguments of $\hat{\mathrm{R}}_{a b c}$ and $\check{\mathrm{R}}_{a b c}$ are the same. Therefore, moving all F's in $\phi_{0}\left(\bar{W}^{\prime}(3, N)\right)$ to the right results in the same transformation of these arguments as moving all $\mathrm{F}^{\prime} \mathrm{s}$ in $\phi_{0}\left(W^{\prime}(3, N)\right)$ to the left.

The y's arguments of $\hat{\mathrm{R}}_{a b c}$ are transformed only by those F's that enter the $\phi_{0}\left(\left\lfloor\bar{W}^{\prime}(2, c-1)\right\rfloor_{c}\right)$ part. Specifically, $\mathrm{y}_{a b}$ does not change, whereas $\mathrm{y}_{a c}$ is transformed by consecutive action of pairs $\mathrm{F}_{a+k, b+1+k, c} \mathrm{~F}_{a+1+k, b+1+k, c}, 0 \leq k \leq c-b-1$, which yields the product of y's in (A.33).

It remains to pull $F_{N}$ in (A.32) to the left. Note that $\mathrm{F}_{N}^{2}=1$. Using (42) and (43), one can verify that

$$
\mathrm{F}_{N} \mathbf{x}_{a b} \mathrm{~F}_{N}=\mathbf{x}_{b-a, b} \prod_{k=1}^{N-b} \frac{\mathbf{x}_{b-a, b+k}}{\mathbf{x}_{b-a+1, b+k}}, \quad \mathrm{~F}_{N} \mathrm{y}_{a b} \mathrm{~F}_{N}=\prod_{k=1}^{b-a} \frac{\mathbf{y}_{k, a+k}}{\mathbf{y}_{k, a+k-1}}
$$

whence

$$
\frac{\mathrm{x}_{a b}}{\mathrm{x}_{a+1, b+1}} \mathrm{~F}_{N}=\mathrm{F}_{N} \frac{\mathrm{x}_{b-a, b}}{\mathrm{x}_{b-a+1, b+1}}, \quad \frac{\mathrm{y}_{a b}}{\mathrm{y}_{a, b+1}} \mathrm{~F}_{N}=\mathrm{F}_{N} \frac{\mathrm{y}_{b-a+1, b}}{\mathrm{y}_{b-a+1, b+1}}
$$

Applying these relations to (A.33), we obtain (A.27). 
Proof of Lemma 2.17. Using (14), (32), and (45), we find

$$
\tau\left(\mathbb{R}_{a b c}\right)=\varphi\left(R_{a b c}\right), \quad \tau\left(\mu_{1}\left(\mathbb{R}_{a b c}\right)\right)=\varphi^{\prime}\left(R_{a b c}\right) .
$$

Therefore, invoking Lemma 2.7, we conclude that

$$
\begin{gathered}
\tau\left(\mathrm{T}_{N}\right)=\tau\left(\left(W^{\prime}(3, N+1)\right)^{\star}\right)=\varphi\left(W^{\prime}(3, N+1)\right), \\
\tau\left(\mu_{1}\left(\mathrm{~T}_{N}\right)\right)=\tau\left(\mu_{1}\left(\left(W^{\prime}(3, N+1)\right)^{\star}\right)\right)=\varphi^{\prime}\left(\bar{W}^{\prime}(3, N+1)\right) .
\end{gathered}
$$

These relations along with Lemma A.3 yield the statement of Lemma 2.17.

\section{References}

[1] Berenstein, A. and A. Zelevinsky. "Quantum cluster algebras." Advances in Mathematics 195, no. 2 (2005): 405-55.

[2] Dimakis, A. and F. Müller-Hoissen. "KP solitons, higher Bruhat and Tamari orders." Progress in Mathematics 299 (2012): 391-423.

[3] Faddeev, L. D. and R. M. Kashaev. “Quantum dilogarithm." Modern Physics Letters A9, no. 5 (1994): 427-34.

[4] Faddeev, L. D. and A. Yu. Volkov. "Abelian current algebra and the Virasoro algebra on the lattice." Physics Letters B315, no. 3-4 (1993): 311-8.

[5] Fock, V. V. and A. B. Goncharov. "The quantum dilogarithm and representations of quantum cluster varieties." Inventiones Mathematicae 175, no. 2 (2009): 223-86.

[6] Frenkel, I. and G. Moore. "Simplex equations and their solutions." Communications in Mathematical Physics 138, no. 2 (1991): 259-71.

[7] Inoue, R., O. Iyama, B. Keller, A. Kuniba, and T. Nakanishi. "Periodicities of T and Y-systems, dilogarithm identities, and cluster algebras II: types $C_{r}, F_{4}$, and $G_{2}$." Publications of the Research Institute for Mathematical Sciences 49, no. 1 (2013): 43-85.

[8] Kashaev, R. M. and T. Nakanishi. "Classical and quantum dilogarithm identities." Symmetry, Integrability and Geometry: Methods and Applications 7 (2011): 102.

[9] Kashaev, R. M. and A. Yu. Volkov. "From the Tetrahedron Equation to Universal R-Matrices." L.D. Faddeev's Seminar on Mathematical Physics, 79-89. American Mathematical Society Translations, Series 2, 201. Providence, RI: American Mathematical Society, 2000.

[10] Keller, B. “On Cluster Theory and Quantum Dilogarithm Identities." Representations of Algebras and Related Topics, 85-116. EMS Series of Congress Reports, Zürich: European Mathematical Society, 2011.

[11] Nakanishi, T. "Periodicities in Cluster Algebras and Dilogarithm Identities." Representations of Algebras and Related Topics, 407-44. EMS Series of Congress Reports, Zürich: European Mathematical Society, 2011.

[12] Schützenberger, M. P. “Une interprétation de certaines solutions de l'équation fonctionnelle: $F(x+y)=F(x) F(y) . "$ Comptes rendus de l'Académie des Sciences 236 (1953): 352-3. 
1100 A. Bytsko and A. Volkov

[13] Sergeev, S. M. “Two-dimensional R-matrices-descendants of three-dimensional Rmatrices." Modern Physics Letters A12, no. 19 (1997): 1393-410.

[14] Volkov, A. Yu. "Pentagon identity revisited." International Mathematics Research Notices 2012, no. 20 (2012): 4619-24.

[15] Zamolodchikov, A. B. "Tetrahedron equations and the relativistic S-matrix of straightstrings in 2+1-dimensions." Communications in Mathematical Physics 79, no. 4 (1981): 489-505. 